\title{
PARP inhibitors in ovarian cancer: evidence for maintenance and treatment strategies
}

\author{
Antonio Bahena-González ${ }^{1,2}$, Alfredo Toledo-Leyva ${ }^{2}$, Dolores Gallardo-Rincón ${ }^{1,2}$ \\ ${ }^{1}$ Medical Oncology Department, Instituto Nacional de Cancerología, Mexico City, Mexico; ${ }^{2}$ Ovarian Cancer Program, Instituto Nacional de \\ Cancerología, Mexico City, Mexico \\ Contributions: (I) Conception and design: A Bahena-González; (II) Administrative support: D Gallardo-Rincón; (III) Provision of study materials or \\ patients: None; (IV) Collection and assembly of data: All authors; (V) Data analysis and interpretation: None; (VI) Manuscript writing: All authors; \\ (VII) Final approval of manuscript: All authors. \\ Correspondence to: Dolores Gallardo-Rincón. San Fernando 22 Sección XVI, Tlalpan, Ciudad de México, 14080, México. \\ Email: dgallardorincon@gmail.com.
}

\begin{abstract}
Ovarian cancer is the most lethal gynecologic malignancy. The long-established primary treatment for ovarian cancer consisted of surgical cytoreduction followed by platinum-based chemotherapy. Unfortunately, this therapeutic approach is related to a high frequency of early relapses. Further chemotherapy is necessary for recurrent disease, but very few patients can be cured. Poly (ADP-ribose) polymerase (PARP) is a family of proteins involved in various DNA repair activities. PARP inhibition leads to synthetic lethality in BRCA mutated or homologous recombination deficient tumors. The development of PARP inhibitors has changed the way ovarian cancer patients are treated. Olaparib, niraparib and rucaparib are orally active and have demonstrated efficacy for both maintenance and treatment settings. These three drugs have gained regulatory approval for different clinical circumstances. They have an acceptable toxicity profile and are generally well tolerated. Common class toxicities include hematologic effects, gastrointestinal effects and fatigue. Moreover, new treatment strategies that combine PARP inhibitors with other drugs, such as angiogenic agents, are being explored. The purpose of this review is to describe the evidence that define the current clinical role of PARP inhibitors in ovarian cancer. The implementation of rationally designed new clinical trials will be crucial to facilitate the best selection of patients and to continue improving clinical outcomes.
\end{abstract}

Keywords: Poly (ADP-ribose) polymerase (PARP); poly (ADP-ribose) polymerase inhibitors; BRCA genes; ovarian cancer

Submitted Feb 08, 2020. Accepted for publication Jul 06, 2020.

doi: $10.21037 /$ cco-20-69

View this article at: http://dx.doi.org/10.21037/cco-20-69

\section{Introduction}

Eukaryotic cells have evolutionarily developed various mechanisms that ensure the integrity of their genome in order to survive. These processes are known as DNA damage response mechanisms (1). Physical, chemical and biological agents can weaken the integrity of the DNA, generating ruptures of one (single-strand breaks, SSB) or both (double-strand breaks, DSB) chains. The main mechanisms for SSB repair are repair by base excision
(BER) and DNA mismatch (MMR) repair (2). When the lesions are more extensive and manage to generate DSB, homologous recombination (HR) repair route, which has a high degree of fidelity in error repair, is used. There are other ways of salvage DSB reparation, such as the nonhomologous end joining (NHEJ), however, its level of repair fidelity is low and produces errors that in the end can result in a significant commitment in genomic stability and therefore in cell survival.

The canonical mechanisms of DNA damage repair (BER 
and HR) depend on the formation of macromolecular complexes that group various effector proteins that ensure the correct execution of the damage repair. Among these are the BRCA1 and BRCA2 proteins, which are associated with proteins such as RAD50, RAD51, NBS1, MRE11, ATM, ATR and CHK2 (3).

For recent years, interest in Poly (ADP-ribose) polymerase (PARP) proteins has been focused due to their key participation in the repair processes of both SSB and DSB (4). PARP enzymes come from a family of 17 members that include PARP1, PARP2, PARP3, PARP5a and PARP5b. PARP1 is the most studied enzyme in the family, given its particular PARylation function, which is a post-translational modification consisting of the addition of ribose poly-ADP to nuclear proteins. PARP detects DNA damage and aids to choice of repair pathway (5). As explained above, in the context of a deficiency in the canonical repair pathways (e.g., failure of the BRCA function), alternative repair pathways are activated. At this point, the activity of the PARP becomes more important for the organism.

A mutation in the BRCA gene that limits its function compromises the functionality of HR repair. As NHEJ repair is activated, function of PARP1 becomes critical. Without this enzyme, the repair process is highly affected, causing the accumulation of genetic errors, which results in a functional incompatibility of the cell and initiating the process of programmed cell death.

Over $15 \%$ of patients with high-grade serous ovarian cancer carry a germline mutation in BRCA1 or BRCA2 (6). PARP inhibitors are synthetic lethal in the presence of $B R C A$ dysfunction and DNA damage. Two articles of preclinical models showed that $B R C A$ mutant cells are highly sensitive to PARP inhibitors $(7,8)$. These agents have been studied in different ovarian cancer populations that include germline $B R C A$-mutated, somatic $B R C A$-mutated, HR deficient and $B R C A$ wild-type patients.

\section{Studies of PARP inhibitors for ovarian cancer}

\section{Olaparib}

The first evidence of PARP inhibition and synthetic lethality in patients with different types of refractory solid tumors was established in a phase I trial that included a pharmacokinetic and pharmacodynamic analysis of olaparib (9). Selection of patients sought to include a $B R C A$ mutation carriers enriched population. Sixty patients were enrolled and 22 were carriers of a $B R C A$ mutation. Olaparib was well tolerated and antitumor activity was reported in mutation carriers with ovarian, breast or prostate cancer. To further evaluate this activity, this study was expanded to evaluate olaparib in a cohort of $B R C A$ mutation carriers with advanced ovarian cancer and differing platinum-free intervals (10). Fifty patients were treated, 13 had platinumsensitive recurrent disease, 24 had platinum-resistant disease and 13 had platinum-refractory disease. Platinumfree interval was found to be associated with response to olaparib, but antitumor activity in platinum-refractory and resistant disease was also observed.

Given the promising results of olaparib in the previous studies, there was great interest in defining the best way to incorporate it into clinical practice. A phase II, multicenter, open-label, randomized study of ovarian cancer patients that recurred within 12 months of completion of platinum therapy and had a BRCA germline mutation, was carried out (11). The study evaluated twicedaily continuous olaparib at doses of 200 or $400 \mathrm{mg}$ versus pegylated liposomal doxorubicin $50 \mathrm{mg} / \mathrm{m}^{2}$ intravenously every 4 weeks. Ninety-seven patients were assigned to the treatment groups. No difference was observed in the end points of PFS or overall response rate (ORR), but the ORR for olaparib $400 \mathrm{mg}$ (31\%) was consistent with previous reports. A possible imbalance between olaparib and PLD arms may have led to an underestimation of olaparib benefit.

Olaparib treatment was first approved in the United States under the provisions of accelerated approval in 2014. The decision was based on another phase II, multicenter study that evaluated the efficacy and safety of olaparib in a spectrum of $B R C A$ mutation associated tumors, including 193 patients with heavily pretreated ovarian cancer, 148 (77\%) of those with a germline mutation in BRCA gene (12). All patients were considered to be platinum-resistant or not candidates to receive platinum-based therapy. In the ovarian cancer cohort, the tumor response rate was $31.1 \%$, median PFS was 7 months and median OS was 16.6 months. Responses to olaparib were observed across different cancer types, supporting the hypothesis that therapy directed against $B R C A$ deficient cells has activity regardless of anatomic organ of origin. Afterwards, the efficacy and safety of olaparib was confirmed in a subgroup of patients with germline $B R C A$ mutated ovarian cancer who had received at least 3 prior lines of chemotherapy (13) (Table 1). The FDA approval of olaparib as monotherapy is in germline $B R C A$ mutated advanced ovarian cancer after having received 3 or more lines of prior chemotherapy. After these results, the 
Table 1 Results of trials that evaluate PARP inhibitors monotherapy strategies in patients with a BRCA mutation

\begin{tabular}{|c|c|c|c|c|c|c|c|c|c|c|}
\hline Trial & Design & Drug & Setting & $\mathrm{N}$ & $\begin{array}{l}\mathrm{mBRCA} \\
\text { required }\end{array}$ & ORR & \multicolumn{2}{|c|}{ mPFS } & $-H R$ & $\mathrm{P}$ \\
\hline Study $42(13)^{a}$ & Phase II & Olaparib & PR & 193 & Yes & $34 \%$ & 6.7 & - & - & - \\
\hline Study 10/ ARIEL $2(14)^{b}$ & Phase II & Rucaparib & PSR, PR & 106 & Yes $^{\mathrm{c}}$ & $53.8 \%$ & 10 & - & - & - \\
\hline QUADRA $(15)^{d}$ & Phase II & Niraparib & PSR, PR & 63 & No & $29 \%$ & - & - & - & - \\
\hline
\end{tabular}

${ }^{a}$, results from patients with resistant ovarian cancer who had received $>3$ prior lines of chemotherapy (154/298); ${ }^{b}$, based on analysis of an integrated efficacy population with patients from Study 10 (part 2A) and ARIEL2 (parts 1 and 2). Eligible patients had ovarian cancer and a $B R C A$ mutation and received at least two prior chemotherapies; ${ }^{c}$, germline mutation in Study 10 ; germline or somatic mutation in ARIEL2; ' , results from BRCA-mutated patients regardless of number of previous lines of therapy and platinum status (63/463); ${ }^{e}$, by blinded independent central review. HR, hazard ratio; mBRCA, BRCA gene mutation; mPFS, median progression-free survival; PARPi, PARP inhibitors; PR, platinum resistant; PSR, platinum sensitive recurrence.

Table 2 Results of trials that evaluate PARP inhibitors maintenance strategies in patients with a $B R C A$ mutation

\begin{tabular}{|c|c|c|c|c|c|c|c|c|c|}
\hline Trial & Design & Drug & Setting & $\mathrm{N}$ & $\begin{array}{l}\mathrm{mBRCA} \\
\text { required }\end{array}$ & \multicolumn{2}{|c|}{ mPFS } & $\mathrm{HR}$ & $P$ \\
\hline Study $19(18,19)^{a}$ & Phase II & Olaparib & PSR & 136 & No & 11.2 & 4.3 & 0.18 & $<0.0001$ \\
\hline SOLO2 (20) & Phase III & Olaparib & PSR & 295 & Yes & 19.1 & 5.5 & 0.30 & $<0.001$ \\
\hline $\operatorname{NOVA}(21)^{b}$ & Phase III & Niraparib & PSR & 203 & No & 21.0 & 5.5 & 0.27 & $<0.001$ \\
\hline SOLO1 (23) ${ }^{d}$ & Phase III & Olaparib & Frontline & 391 & Yes & 49.9 & 13.8 & 0.31 & $<0.001$ \\
\hline PRIMA (24) & Phase III & Niraparib & Frontline & 223 & No & 22.1 & 10.9 & 0.40 & $<0.001$ \\
\hline
\end{tabular}

${ }^{\mathrm{a}}$, results from patients with a BRCA mutation (136/265); ${ }^{\mathrm{b}}$, results from the gBRCA cohort $\left(203 / 553\right.$ patients); ${ }^{\mathrm{c}}$, results from patients with a BRCA mutation (196/564); ${ }^{d}$, based on PFS sensitivity analysis to assess possible attrition bias; ${ }^{e}$, results from the $B R C A$ mutation subgroup (223/733). HR, hazard ratio; mBRCA, BRCA gene mutation; mPFS, median progression-free survival; PARPi, PARP inhibitors; $\mathrm{PR}$, platinum resistant; PSR, platinum sensitive recurrence.

SOLO3 trial was designed as a phase III confirmatory trial to demonstrate a positive result versus chemotherapy in advanced ovarian cancer.

To further evaluate the efficacy and safety of olaparib in women with recurrent ovarian cancer and a documented germline BRCA mutation, a pooled analysis of two phase I and four phase II trials, including the previously cited trials, was published (17). Of the 300 patients, 205 (75\%) had received $\geq 3$ lines of prior chemotherapy. Treatment with olaparib was associated with an ORR of $36 \%$ and a median duration of response of 7.4 months. Treatment with olaparib had an acceptable and manageable safety profile and most important adverse events were tolerable nausea and fatigue.

The second olaparib FDA approval as a maintenance therapy for recurrent, platinum-sensitive disease was based on the results of Study 19, a phase II, doubleblind, placebo-controlled, randomized trial enrolling 265 patients who were clinically enriched for PARP inhibitor treatment response markers (i.e., women with relapsed, platinum-sensitive, high-grade serous ovarian cancer) and showed a response to their most recent platinum-based chemotherapy (18) (Table 2). Patients were assigned to receive olaparib capsules, at a dose of $400 \mathrm{mg}$ twice daily or placebo. After 153 events, the analysis showed that PFS was 3.6 months longer in the olaparib group (from 4.8 to 8.4 months) and the toxicity profile was manageable. A pre-planned, retrospective analysis demonstrated that BRCA mutated patients obtained the greatest benefit from the PARP inhibitor (HR 0.18, $\mathrm{P}<0.0001$ ), however, 
an advantage was also seen for $B R C A$ wild type treated patients (HR 0.54, $\mathrm{P}<0.0075)(19)$. Long term efficacy and safety analysis showed an apparent OS advantage irrespective of $B R C A$ mutation and a low incidence of discontinuations due to adverse events (6\%) (25).

The efficacy of maintenance treatment with olaparib in platinum-sensitive recurrent ovarian cancer was confirmed in the SOLO2 trial, a phase III, double-blind, placebo-controlled trial of 295 patients with germline $B R C A$ mutated ovarian cancer that had received $\geq 2$ prior platinum-based chemotherapy regimens and had a complete or partial response (20). Patients were randomized to either a new tablet formulation of olaparib (two $150 \mathrm{mg}$ tablets taken orally, twice daily) or placebo until progression. The hazard ratio for PFS was $0.30(\mathrm{P}<0.0001)$, corresponding to a 13.6-month improvement (from 5.5 to 19.1 months). Olaparib maintenance was also related to a significant improvement in time to second progression, time to first, and time to second subsequent therapies. The most common grade $1-2$ events reported in $\geq 20 \%$ in patients that received olaparib were nausea $(73 \%)$, fatigue or asthenia (62\%), vomiting (35\%), diarrhea (32\%) dysgeusia (27\%), headache $(25 \%)$, anemia (24\%), abdominal pain (22\%), hyporexia (22\%) and constipation (21\%). Grade 1-2 neutropenia and thrombocytopenia occurred in $14 \%$ and $13 \%$ of patients. The incidence of grade 3-4 events was low. The only grade 3-4 event with an incidence of $\geq 10 \%$ was anemia (19\%). Furthermore, health-related quality of life was not affected while on treatment (26). The new tablet formulation reduces the treatment burden from 16 capsules to four tables per day.

The SOLO1 trial was conducted to evaluate the efficacy of maintenance treatment with olaparib tablets in women with newly diagnosed stage III or IV ovarian cancer, with a mutation in $B R C A$, and that obtained a response after first line platinum-based chemotherapy (23). The SOLO1 randomized, double-blind, placebo-controlled trial was conducted in 391 ovarian cancer patients. The use of maintenance olaparib provided a substantial PFS benefit, with a $70 \%$ lower risk of progression (HR 0.30; $\mathrm{P}<0.001$ ), corresponding to a median PFS of 13.8 months in the placebo arm and a not reached median with olaparib after nearly 3 years of follow-up. Significant increases in time to first subsequent therapy and time to second disease progression were also noted with olaparib. Although most patients in this trial had a germline BRCA mutation, the results with other PARP inhibitors suggest that the findings could be expanded to patients with a somatic
$B R C A$ mutation (22). This led to the FDA approval for the maintenance treatment of patients with deleterious or suspected deleterious germline or somatic BRCA mutated advanced ovarian cancer who obtained a response (complete or partial) after platinum-based chemotherapy.

The SOLO3 study was presented at the 2019 American Society of Clinical Oncology (ASCO) Annual Meeting (16). This study randomly assigned 178 patients with platinumsensitive, $B R C A$ mutated recurrent ovarian cancer in order to confirm a benefit from treatment with olaparib monotherapy compared with physician's choice chemotherapy treatment (27). ORR for olaparib by blinded independent review was $72 \%$ in contrast with $51 \%$ in the chemotherapy arm (OR 2.53; $\mathrm{P}=0.002)$. In addition, olaparib derived a $38 \%$ progression risk decline compared with chemotherapy (HR, 0.62) and a median PFS benefit of 4.2 months (13.4 vs. 9.2 months; $\mathrm{P}=0.13$ ). In accordance with these results, chemotherapy-free treatment may be a reasonable treatment option for women with $B R C A$ mutation and platinum-sensitive relapsed ovarian cancer.

\section{Niraparib}

Niraparib is a PARP inhibitor that demonstrated inhibition of tumor growth in preclinical models with loss of $B R C A$ function (28). A two-part, phase I dose-escalation study of niraparib was undertaken in human carriers of $B R C A$ mutations and ovarian and prostate cancers, in order to assess pharmacokinetic and pharmacodynamic profiles, as well as antitumor activity (29). Patients were not suitable to receive any established treatment. A total of 100 patients were enrolled and 49 had ovarian cancer. The maximum tolerated dose was $300 \mathrm{mg} /$ day. Antitumor activity was achieved in both mutated and wild type $B R C A$ patients. Responses were documented in 8 of $20(40 \%) B R C A$ mutated ovarian cancer patients. Out of 3 patients with wild-type $B R C A$, platinum-sensitive ovarian cancer, 2 (67\%) achieved durable partial responses by RECIST or CA 125 or both. The use of niraparib was associated with a low frequency of serious adverse events.

The NOVA study was a phase III, randomized, doubleblind trial that sought to evaluate the efficacy and safety of niraparib (300 $\mathrm{mg}$ orally daily) as maintenance treatment for patients with platinum-sensitive, relapsed ovarian cancer (21). Patients were eligible regardless of germline $B R C A$ mutation status and were categorized in a germlinemutated (gBRCA) and a non-germline mutated (nongBRCA) cohort. Before the database lock, tissue samples 
were tested to identify the population of patients in the non-gBRCA cohort in whom tumors had homologous recombination deficiency (HRD-positive subgroup). A total of 553 patients were enrolled, 201 in the gBRCA and 345 in the non-gBRCA cohort. In the gBRCA cohort, the median PFS was 21 months in the niraparib group and 5.5 months in the placebo group (HR, 0.72; $\mathrm{P}<0.001)$. A PFS benefit of niraparib was also documented in the nongBRCA cohort (median, 9.3 vs. 3.9 months; HR, 0.45; $\mathrm{P}<0.001$ ) and in the HRD-positive subgroup (median, 12.9 vs. 3.8 months; $\mathrm{HR}, 0.38 ; \mathrm{P}<0.001)$. Bone marrow toxicity was moderate and manageable. The incidence of grade 1-2 thrombocytopenia, anemia and neutropenia in patients with niraparib was $28 \%, 25 \%$ and $11 \%$, respectively. Common non-hematologic grade 1-2 toxicities were nausea $(71 \%)$, fatigue $(51 \%)$, constipation $(39 \%)$, vomiting (32\%), headache (26\%), hyporexia (25\%), insomnia (24\%) and abdominal pain (22\%). Grade 3-4 toxicity was mainly represented by thrombocytopenia (34\%), anemia (25\%) and neutropenia (20\%). Quality of life does not appear to be affected by use of niraparib (30). FDA first approval of niraparib in 2017 was based upon these results and assigned for the maintenance treatment of patients with recurrent ovarian cancer who are in a complete or partial response to platinum-based chemotherapy. Niraparib was the first PARP inhibitor to be approved that does not require $B R C A$ mutation.

The indication of niraparib as monotherapy is based on data from the QUADRA study, a phase II, multicenter, open label, single arm clinical trial that evaluated its activity and safety in ovarian cancer patients who had been treated with three or more previous chemotherapy regimens (15). The primary objective was the ORR in the primary efficacy population (e.g., patients with HR-deficient tumors sensitive to their last platinumbased therapy who had received 3-4 previous regimens). Of a total of 463 patients, 47 were included in the primary efficacy population and gained an ORR of $28 \%$ $(\mathrm{P}=0.00053)$. This led to approval from the FDA for the treatment of advanced ovarian cancer patients who have been treated with three more prior chemotherapy regimens and have an HR-deficiency positive status by either a deleterious or suspected deleterious $B R C A$ mutation or genomic instability, and who have progressed more than 6 months after response to the last platinumbased chemotherapy. This represents the first time a PARP inhibitor has been approved as monotherapy for women with ovarian cancer, regardless or their BRCA mutation status, in the heavily pretreated setting.

To test the activity and safety of niraparib maintenance after frontline treatment, the PRIMA trial was designed and carried out (24). This phase III, randomized, doubleblind trial assigned patients (N=733) with newly diagnosed advanced ovarian cancer to receive niraparib maintenance therapy or placebo after a response to platinum-based chemotherapy. Tumor samples were tested to identify those with HR-deficiency, determined as the presence of a $B R C A$ mutation, a score of $\geq 42$ on the myChoice test, or both. In patients with HR-deficiency the median PFS was 21.9 months with niraparib and 10.4 without niraparib (HR, $0.43 ; \mathrm{P}<0.001)$. Median PFS in the overall population was 13.8 months with niraparib and 8.2 months with placebo (HR, 0.62; $\mathrm{P}<0.001$ ). These results confirmed that benefit of niraparib is present regardless of the presence or absence of HR-deficiency.

\section{Rucaparib}

The effect of rucaparib in humans was first assessed in combination with temozolomide in a phase I trial of patients with different solid tumors (31). In this study, patients received intravenous escalating doses of rucaparib in combination with temozolomide $\left(100 \mathrm{mg} / \mathrm{m}^{2} / \mathrm{d}\right.$ for 5 days every 28 days) to establish the PARP inhibitory dose. The PARP inhibitory dose was $12 \mathrm{mg} / \mathrm{m}^{2}$ on the basis of a $74 \%$ to $97 \%$ inhibition of peripheral blood lymphocytes PARP activity.

A phase II study investigated different schedules and dose levels of rucaparib in patients with germline $B R C A$ mutant breast or ovarian cancers (32). This study provided evidence that continuous dosing of oral rucaparib is required for optimal response and derives a greater benefit than intermittent intravenous dosing. After these observations, recruitment to the intravenous formulation cohorts was discontinued. However, the maximum oral dose of rucaparib was not sufficiently evaluated.

Study 10 was a three-part, phase I-II trial to evaluate efficacy, toxicity, and the recommended phase II dose based on the maximum tolerate dose (33). Part 1 (phase I of dose escalation) included 56 women with breast or ovarian cancer with or without a $B R C A$ mutation. No maximum tolerated dose was identified and the $600 \mathrm{mg}$ twice-daily was selected as a recommended phase II dose. Part 2A (phase II expansion) assessed the efficacy of rucaparib in women with platinum-sensitive recurrent ovarian cancer with a germline BRCA mutation. Of 42 patients, $25(59.6 \%)$ achieved a 
RECIST response. Rucaparib showed a manageable safety profile.

The ARIEL2 trial was a two-part, single arm, phase II trial designed to assess efficacy and safety of rucaparib. In part 1, participants $(\mathrm{N}=204)$ had ovarian cancer and at least one previous platinum therapy (34). They were classified into one of three predefined HR-deficiency subgroups: $B R C A$ mutant (germline or somatic), BRCA wild-type and loss of heterozygosity ( $\mathrm{LOH}$ ) high, and $B R C A$ wild-type and LOH low. Median PFS was 12.8, 5.7 and 5.2 months for BRCA mutant, BRCA wild-type and $\mathrm{LOH}$ high, and BRCA wild-type and LOH low subgroups. These outcomes suggest that assessment of LOH status can be used as a reliable biomarker. Part 2 is an extension that includes ovarian tumors that are platinum-sensitive, platinumresistant, or platinum-refractory and received 3-4 prior chemotherapies. Results from ARIEL2 part 2 are still pending.

The efficacy of oral rucaparib at a dose of $600 \mathrm{mg}$ twice daily was also evaluated in a combined analysis of patients from Study 10 (part 2A) and ARIEL2 (parts 1 and 2) who had ovarian cancer, received $\geq 2$ prior lines of chemotherapy and had a deleterious germline or somatic $B R C A$ mutation ( $\mathrm{N}=106)$ (14). This population obtained an ORR of $53.8 \%$, a $34 \%$ rate of stable disease and a manageable safety profile. The results of the pooled analysis of these two phase II trials (Study 10 and ARIEL2) led to the accelerated FDA approval of rucaparib for the treatment of patients with ovarian cancer associated with a deleterious germline or somatic $B R C A$ mutation who have received $\geq 2$ lines of chemotherapy.

Rucaparib has also proved effective as a maintenance treatment option for platinum-sensitive relapsed ovarian cancer. The ARIEL3 study was a phase III, randomized, double-blind, placebo controlled trial including 564 patients with ovarian cancer who had received at least two previous platinum-based chemotherapy regimens and achieved a response to their last platinum-based regimen (22). The $B R C A$ mutation status and the size of residual disease were not restricted. Patients were assigned to a rucaparib $600 \mathrm{mg}$ twice daily arm or a placebo arm. They were categorized into three cohorts: patients with germline or somatic $B R C A$ mutations, patients with HR-deficiencies (high loss of heterozygosity), and the intention-to-treat population. Median PFS in BRCA mutation carriers was 16.6 months in the rucaparib group and 5.4 months in the placebo group ( $\mathrm{HR}, 0.23 ; \mathrm{P}<0.0001)$. In patients with an $\mathrm{HR}$ deficiency it was 13.6 and 5.4 months (HR, 0.32; $\mathrm{P}<0.0001)$.
In the intention-to-treat population, it was 10.8 and 5.4 months (HR, 0.36; $\mathrm{P}<0.0001)$. Grade $1-2$ hematologic toxicities included thrombocytopenia (23\%), anemia (19\%) and neutropenia (18\%). Common non-hematologic grade $1-2$ toxicities that occurred across $\geq 20 \%$ of patients that received rucaparib were nausea ( $72 \%)$, fatigue (asthenia) $(63 \%)$, dysgeusia (39\%), constipation (35\%), vomiting $(33 \%)$, diarrhea (31\%), abdominal pain (27\%) and hyporexia $(23 \%)$ and increase in aminotransferase concentrations (23\%). The most common treatment-emergent adverse events of grade $3-4$ were anemia (19\%) and increase in aminotransferase concentrations (10\%).

\section{Combination with angiogenesis inhibitors}

Since the introduction of bevacizumab, angiogenesis inhibitors represent a treatment option for epithelial ovarian cancer. Data from phase III trials confirming bevacizumab and cediranib benefit in ovarian cancer have been published (35-38). Moreover, hypoxia has been shown to be associated with impaired homologous recombination, and repression of $B R C A$ expression by hypoxia is a mechanism of $B R C A$ inactivation in the absence of a genetic mutation (39). In light of these observations, one can hypothesize that PARP inhibitors may be particularly effective against hypoxic cancer cells in which BRCA expression is down-regulated by anti-angiogenic drugs.

Molecular therapy combination of olaparib and cediranib was evaluated in a randomized, phase II trial that assessed efficacy and safety in women with platinum-sensitive ovarian cancer $(40,41)$. This trial included 90 patients who were randomly assigned to olaparib or olaparib with cediranib. The addition of cediranib led to a more than 8 months PFS improvement (17.7 and 9 months; HR, 0.42), and a greater frequency of serious fatigue, diarrhea and hypertension. A post-hoc analysis stratified patients by their germline $B R C A$ status and suggested that women who were wild-type or not known to have a BRCA mutation derived a greater PFS benefit (16.5 vs. 5.7 months; HR, 0.32; $\mathrm{P}=0.008$ ) compared to those with a $B R C A$ mutation (19.4 vs. 16.5 months; HR, $0.55 ; \mathrm{P}=0.16$ ), although the latter group was small. A greater synergism with the combination in the setting of more HRproficient tumours or hypoxia could explain this difference.

The AVANOVA2 phase II study was designed to investigate the effect and safety of the chemotherapy-free combination treatment with niraparib and bevacizumab (42). This trial randomized 97 patients to receive oral niraparib $300 \mathrm{mg}$ daily plus bevacizumab $15 \mathrm{mg} / \mathrm{kg}$ every 3 weeks 
or single-agent niraparib. Eligible patients had to have previously received platinum-containing therapy for primary disease but $\leq 1$ prior non-platinum-containing regimen for recurrent disease. $B R C A$ mutation was not mandatory and patients were stratified by HR-deficiency status. As part of the subgroup analyses, the combination therapy also improved PFS in patients with and without HR-deficiency. The safety analysis was consistent with the known profiles of both drugs combined.

The phase III trial PAOLA-1 evaluated maintenance therapy with the combination of olaparib and bevacizumab compared with bevacizumab and placebo after frontline treatment of patients who were receiving chemotherapy plus bevacizumab, regardless of $B R C A$ mutation status (43). A total of 806 patients were enrolled. The median PFS was longer in the olaparib group (22.1 vs. 16.6 months; HR 0.59; $\mathrm{P}<0.001)$, with a greater benefit in patients with a $B R C A$ mutation and with HR-deficient tumors. The addition of olaparib did not increase the known toxic effects of bevacizumab and there was no difference in health-related quality of life between the studied groups. The combination improved PFS compared with olaparib (median $11.9 \mathrm{vs}$. 5.5 months; HR, 0.35; $\mathrm{P}<0.0001)$.

\section{Toxicities}

The toxicity of PARP inhibitors has been widely described in a recently published review (44). In general, the toxicity profiles of olaparib, niraparib and rucaparib are similar and manageable. Adverse events are usually managed with appropriate delays and dose modifications. Frequency of adverse events leading to olaparib dose interruptions in SOLO2 study was $45 \%$ compared to $18 \%$ in the placebo group (20). Olaparib dose reductions following adverse events were $25 \%$ vs. $3 \%$. Discontinuations owing to adverse events were $11 \%$ and $2 \%$. In the NOVA trial, niraparib dose interruptions were $68.9 \%$, dose reductions were $66.5 \%$ and discontinuations were $14.7 \%$ (21). Rucaparib treatment interruptions due to an adverse event occurred in $64 \%$ of patients of the ARIEL3 trial, dose reductions in $55 \%$ and discontinuations in $13 \%$ (22).

PARP inhibitors class adverse events include hematologic effects, gastrointestinal effects and fatigue (Table 3). Hematologic toxicities typical occur during the first cycles of treatment and are usually transient. Anemia is the prominent hematologic toxicity among PARP inhibitors. Anemia of all grades occurred in $44 \%$ of patients that received olaparib in the $\mathrm{SOLO} 2$ trial, in $50 \%$ of patients treated with niraparib in the NOVA trial, and in $37 \%$ that received rucaparib in ARIEL3. Grade 3-4 anemia was documented in $19 \%$ of patients with olaparib, $25 \%$ of patients with niraparib, and $19 \%$ in patients with rucaparib. Thrombocytopenia is the main cause of niraparib hematologic toxicity, occurring in $61 \%$ of patients in any grade and in $34 \%$ of patients in grades $3-4$. Neutropenia is also more prominent in patients with niraparib.

The most common gastrointestinal toxicity is nausea. Nausea of all grades was documented in $76 \%$ of patients with olaparib, $74 \%$ of patients with niraparib and $75 \%$ of patients with rucaparib but grade 3-4 nausea is not common in patients with PARP inhibitors. In most cases, nausea responds to standard oral antiemetics such as metoclopramide. Patients initiating a PARP inhibitor could receive antiemesis primary or secondary prophylaxis to help prevent nausea or vomiting. It should be noted that CYP 3A4/5 enzymes are related to the metabolic clearance of olaparib and concomitant administration with inducers of inhibitors of CYP3A4/5 isoenzymes should be done very cautiously. Neurokinin 1 receptor antagonist aprepitant is a CYP3A inhibitor and should be avoided (45). Other gastrointestinal effects include vomiting, constipation, diarrhea, dysgeusia, dyspepsia, stomatitis, and hyporexia. Rucaparib is associated with grade 3-4 elevation of hepatic enzymes (10\%), but this effect is transient and self-limiting (22).

Fatigue is another common symptom related to the use of PARP inhibitors. It has been related to $66 \%$ of patients using olaparib, $59 \%$ of patients with niraparib, and $69 \%$ of patients with rucaparib, but grades $3-4$ presentation is not common. Some experts suggest that patients should be reassured about the frequency of this symptom and its transitory nature before initiating the drug (46). In addition, patient should receive guidance on undertaking moderate exercise and ensuring adequate nutrition.

\section{Conclusions and future directions}

For many years, cytoreductive surgery and chemotherapy were the only treatment options for ovarian cancer. The addition of antiangiogenic therapy improved patient outcomes, but no clear effect on survival has been demonstrated. The recent addition of PARP inhibitors to the systemic treatment options has clearly caused a paradigm shift in ovarian cancer management. Olaparib, niraparib and rucaparib are approved by regulatory agencies for ovarian cancer treatment. As previously mentioned, 
Table 3 Selected adverse events from the platinum sensitive maintenance trials

\begin{tabular}{|c|c|c|c|}
\hline Adverse event & SOLO2 (20) (olaparib) & NOVA (21) (niraparib) & ARIEL3 (22) (rucaparib) \\
\hline \multicolumn{4}{|c|}{ Hematologic events } \\
\hline \multicolumn{4}{|l|}{ Anaemia } \\
\hline All grades & $44 \%$ & $50 \%$ & $37 \%$ \\
\hline Grade 3-4 & $19 \%$ & $25 \%$ & $19 \%$ \\
\hline \multicolumn{4}{|c|}{ Thrombocytopenia } \\
\hline All grades & $14 \%$ & $61 \%$ & $28 \%$ \\
\hline Grade 3-4 & $1 \%$ & $34 \%$ & $5 \%$ \\
\hline \multicolumn{4}{|l|}{ Neutropenia } \\
\hline All grades & $19 \%$ & $30 \%$ & $18 \%$ \\
\hline \multicolumn{4}{|l|}{ Nausea } \\
\hline All grades & $76 \%$ & $74 \%$ & $75 \%$ \\
\hline Grade 3-4 & $3 \%$ & $3 \%$ & $4 \%$ \\
\hline \multicolumn{4}{|l|}{ Vomiting } \\
\hline All grades & $38 \%$ & $34 \%$ & $37 \%$ \\
\hline Grade 3-4 & $3 \%$ & $2 \%$ & $4 \%$ \\
\hline \multicolumn{4}{|c|}{ Decreased appetite } \\
\hline All grades & $22 \%$ & $25 \%$ & $23 \%$ \\
\hline \multicolumn{4}{|l|}{ Diarrhea } \\
\hline All grades & $33 \%$ & 19 & $32 \%$ \\
\hline Grade 3-4 & $1 \%$ & $<1 \%$ & $1 \%$ \\
\hline \multicolumn{4}{|l|}{ Dysgeusia } \\
\hline All grades & $27 \%$ & $10 \%$ & $39 \%$ \\
\hline Grade 3-4 & $0 \%$ & $0 \%$ & $0 \%$ \\
\hline \multicolumn{4}{|l|}{ Dyspepsia } \\
\hline All grades & $11 \%$ & $11 \%$ & $15 \%$ \\
\hline Grade 3-4 & $0 \%$ & $0 \%$ & $<1 \%$ \\
\hline \multicolumn{4}{|l|}{ Miscellaneous } \\
\hline \multicolumn{4}{|l|}{ Fatigue } \\
\hline All grades & $66 \%$ & $59 \%$ & $69 \%$ \\
\hline Grade 3-4 & $4 \%$ & $8 \%$ & $7 \%$ \\
\hline
\end{tabular}

Table 3 (continued) 
Table 3 (continued)

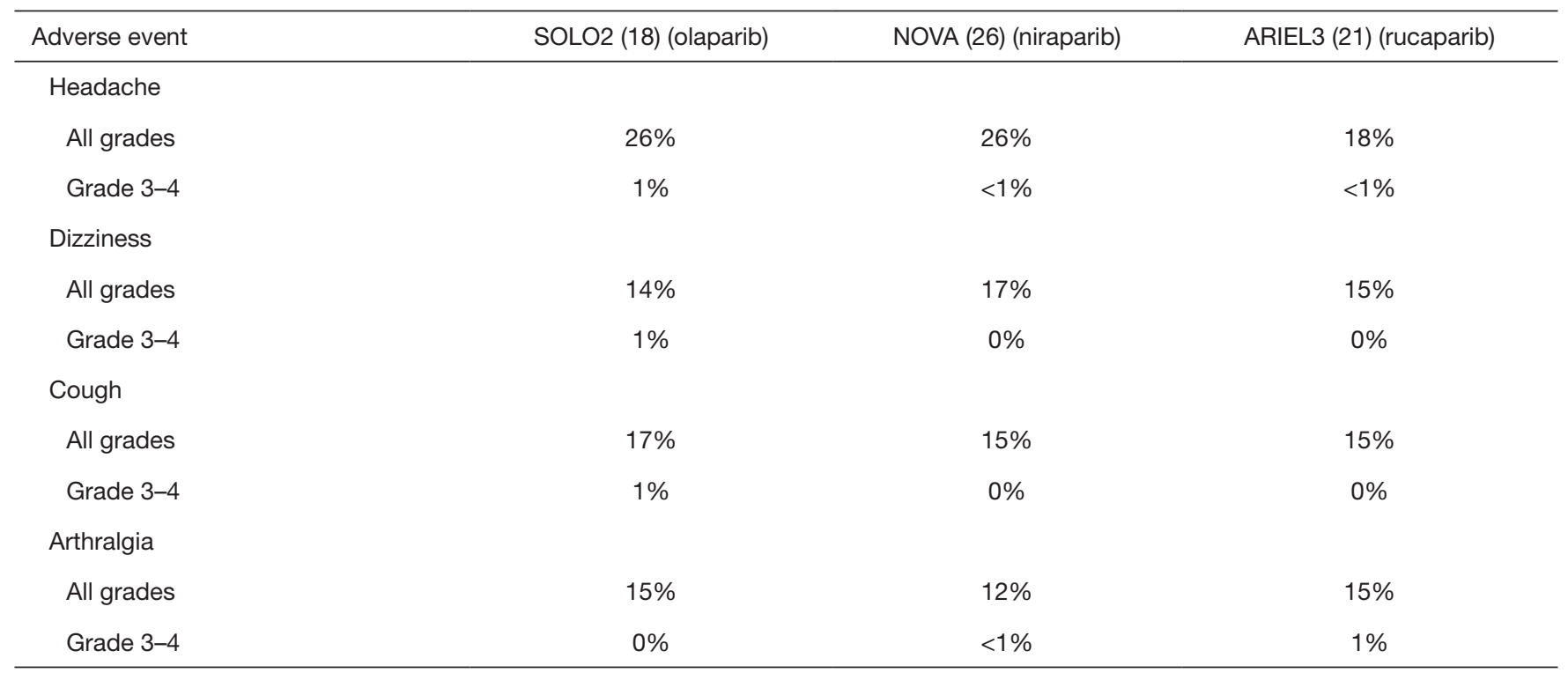

these drugs have been rapidly moving in to the first line setting. Genetic testing for $B R C A$ and HR- deficiency alterations has allowed better personalization of treatment, but selection of the best candidates continues to be a challenge. Many studies evaluating the optimal application of PARP inhibitors in medical practice are in progress.

The combination of PARP inhibitors and immunotherapy is currently under active investigation. Mutations generated after alterations of the DNA damage response mechanisms can originate neoantigens. Neoantigens are optimal targets for immunotherapy. Early phase clinical trials testing the combination of PARP inhibitors plus immunotherapy in ovarian cancer patients have showed some promising results $(47,48)$. At least three phase III studies are evaluating the benefits of this strategy (NCT03602859, NCT03737643 and NCT03522246).

Another issue that needs to be established is the efficacy of retreatment. In patients with recurrence after having received a PARP inhibitor, it is possible that sensitivity to PARP inhibitors might be retained after subsequent lines of cytotoxic treatment. A phase III trial that will try to confirm this assumption is being conducted (NCT03106987).

Other PARP inhibitors are also being tested in ovarian cancer patients (e.g., veliparib, talazoparib). VELIA was the first phase III study that focused on the efficacy and safety of the combination of a PARP inhibitor (veliparib) and chemotherapy (49). Other ongoing trials are exploring this combination strategy.
Most toxicities observed with PARP inhibitors are relatively mild and require only observation or standard supportive care.

\section{Acknowledgments}

Funding: None.

\section{Footnote}

Provenance and Peer Review: This article was commissioned by the Guest Editor (Heriberto Medina-Franco) for the series "Ovarian Cancer" published in Chinese Clinical Oncology. The article was sent for external peer review organized by the Guest Editor and the editorial office.

Conflicts of Interest: All authors have completed the ICMJE uniform disclosure form (available at http://dx.doi. org/10.21037/cco-20-69). The series "Ovarian Cancer" was commissioned by the editorial office without any funding or sponsorship. Dr. ABG reports personal fees from Roche, outside the submitted work. Dr. DGR reports personal fees from Astra Zeneca, from Roche, from Lilly, outside the submitted work. The other author has no other conflicts of interest to declare.

Ethical Statement: The authors are accountable for all aspects of the work in ensuring that questions related 
to the accuracy or integrity of any part of the work are appropriately investigated and resolved.

Open Access Statement: This is an Open Access article distributed in accordance with the Creative Commons Attribution-NonCommercial-NoDerivs 4.0 International License (CC BY-NC-ND 4.0), which permits the noncommercial replication and distribution of the article with the strict proviso that no changes or edits are made and the original work is properly cited (including links to both the formal publication through the relevant DOI and the license). See: https://creativecommons.org/licenses/by-nc-nd/4.0/.

\section{References}

1. Hoeijmakers JHJ. DNA Damage, Aging, and Cancer. N Engl J Med 2009;361:1475-85.

2. Caldecott KW. Single-strand break repair and genetic disease. Nat Rev Genet 2008;9:619-31.

3. Huen MSY, Sy SMH, Chen J. BRCA1 and its toolbox for the maintenance of genome integrity. Nat Rev Mol Cell Biol 2010;11:138-48.

4. Gupte R, Liu Z, Kraus WL. PARPs and ADP-ribosylation: recent advances linking molecular functions to biological outcomes. Genes Dev 2017;31:101-26.

5. Morales JC, Li L, Fattah FJ, et al. Review of poly (ADPribose) polymerase (PARP) mechanisms of action and rationale for targeting in cancer and other diseases. Crit Rev Eukaryot Gene Expr 2014;24:15-28.

6. Pal T, Permuth-Wey J, Betts JA, et al. BRCA1 and BRCA2 mutations account for a large proportion of ovarian carcinoma cases. Cancer 2005;104:2807-16.

7. Bryant HE, Schultz N, Thomas HD, et al. Specific killing of BRCA2-deficient tumours with inhibitors of poly(ADPribose) polymerase. Nature 2005;434:913-7.

8. Farmer H, McCabe H, Lord CJ, et al. Targeting the DNA repair defect in BRCA mutant cells as a therapeutic strategy. Nature 2005;434:917-21.

9. Fong PC, Boss DS, Yap TA, et al. Inhibition of Poly(ADPRibose) Polymerase in Tumors from BRCA Mutation Carriers. N Engl J Med 2009;361:123-34.

10. Fong PC, Yap TA, Boss DS, et al. Poly(ADP)-ribose polymerase inhibition: Frequent durable responses in BRCA carrier ovarian cancer correlating with platinumfree interval. J Clin Oncol 2010;28:2512-9.

11. Kaye SB, Lubinski J, Matulonis U, et al. Phase II, openlabel, randomized, multicenter study comparing the efficacy and safety of olaparib, a poly (ADP-ribose) polymerase inhibitor, and pegylated liposomal doxorubicin in patients with BRCA1 or BRCA2 mutations and recurrent ovarian cancer. J Clin Oncol 2012;30:372-9.

12. Kaufman B, Shapira-Frommer R, Schmutzler RK, et al. Olaparib monotherapy in patients with advanced cancer and a germline BRCA1/2 mutation. J Clin Oncol 2015;33:244-50.

13. Domchek SM, Aghajanian C, Shapira-Frommer R, et al. Efficacy and safety of olaparib monotherapy in germline BRCA1 / 2 mutation carriers with advanced ovarian cancer and three or more lines of prior therapy. Gynecol Oncol 2016;140:199-203.

14. Oza AM, Tinker AV, Oaknin A, et al. Antitumor activity and safety of the PARP inhibitor rucaparib in patients with high-grade ovarian carcinoma and a germline or somatic BRCA1 or BRCA2 mutation: Integrated analysis of data from Study 10 and ARIEL2. Gynecol Oncol 2017;147:267-75.

15. Moore KN, Secord AA, Geller MA, et al. Niraparib monotherapy for late-line treatment of ovarian cancer (QUADRA): a multicentre, open-label, single-arm, phase 2 trial. Lancet Oncol 2019;20:636-48.

16. Penson RT, Villalobos Valencia R, Cibula D, et al. Olaparib monotherapy versus (vs) chemotherapy for germline BRCA-mutated (gBRCAm) platinum-sensitive relapsed ovarian cancer (PSR OC) patients (pts): Phase III SOLO3 trial. J Clin Oncol 2019;37:5506.

17. Matulonis UA, Penson RT, Domchek SM, et al. Olaparib monotherapy in patients with advanced relapsed ovarian cancer and a germline BRCA1/2 mutation: A multistudy analysis of response rates and safety. Ann Oncol 2016;27:1013-9.

18. Ledermann J, Harter P, Gourley C, et al. Olaparib maintenance therapy in platinum-sensitive relapsed ovarian cancer. N Engl J Med 2012;366:1382-92.

19. Ledermann J, Harter P, Gourley C, et al. Olaparib maintenance therapy in patients with platinum-sensitive relapsed serous ovarian cancer: A preplanned retrospective analysis of outcomes by BRCA status in a randomised phase 2 trial. Lancet Oncol 2014;15:852-61.

20. Pujade-Lauraine E, Ledermann JA, Selle F, et al. Olaparib tablets as maintenance therapy in patients with platinumsensitive, relapsed ovarian cancer and a BRCA1/2 mutation (SOLO2/ENGOT-Ov21): a double-blind, randomised, placebo-controlled, phase 3 trial. Lancet Oncol 2017;18:1274-84.

21. Mirza MR, Monk BJ, Herrstedt J, et al. Niraparib maintenance therapy in platinum-sensitive, recurrent 
ovarian cancer. N Engl J Med 2016;375:2154-64.

22. Coleman RL, Oza AM, Lorusso D, et al. Rucaparib maintenance treatment for recurrent ovarian carcinoma after response to platinum therapy (ARIEL3): a randomised, double-blind, placebo-controlled, phase 3 trial. Lancet 2017;390:1949-61.

23. Moore K, Colombo N, Scambia G, et al. Maintenance olaparib in patients with newly diagnosed advanced ovarian cancer. N Engl J Med 2018;379:2495-505.

24. González-Martín A, Pothuri B, Vergote I, et al. Niraparib in Patients with Newly Diagnosed Advanced Ovarian Cancer. N Engl J Med 2019;381:2391-402.

25. Friedlander M, Matulonis U, Gourley C, et al. Longterm efficacy, tolerability and overall survival in patients with platinum-sensitive, recurrent high-grade serous ovarian cancer treated with maintenance olaparib capsules following response to chemotherapy. Br J Cancer 2018;119:1075-85.

26. Ledermann JA, Harter P, Gourley C, et al. Quality of life during olaparib maintenance therapy in platinumsensitive relapsed serous ovarian cancer. Br J Cancer 2016;115:1313-20.

27. Penson RT, Valencia RV, Cibula D, et al. Olaparib Versus Nonplatinum Chemotherapy in Patients With PlatinumSensitive Relapsed Ovarian Cancer and a Germline BRCA1/2 Mutation (SOLO3): A Randomized Phase III Trial. J Clin Oncol 2020;38:1164-74.

28. Jones P, Altamura S, Boueres J, et al. Discovery of 2-\{4-[(3S)-piperidin-3-yl]phenyl\}-2H-indazole-7carboxamide (MK-4827): A novel oral poly(ADP-ribose) polymerase (PARP) inhibitor efficacious in BRCA-1 and -2 mutant tumors. J Med Chem 2009;52:7170-85.

29. Sandhu SK, Schelman WR, Wilding G, et al. The poly(ADP-ribose) polymerase inhibitor niraparib (MK4827) in BRCA mutation carriers and patients with sporadic cancer: A phase 1 dose-escalation trial. Lancet Oncol 2013;14:882-92.

30. del Campo JM, Matulonis UA, Malander S, et al. Niraparib Maintenance Therapy in Patients With Recurrent Ovarian Cancer After a Partial Response to the Last Platinum-Based Chemotherapy in the ENGOTOV16/NOVA Trial. J Clin Oncol 2019;37:2968-73.

31. Plummer R, Middleton M, Wilson R, et al. First in human phase I trial of the PARP inhibitor AG-014699 with temozolomide (TMZ) in patients (pts) with advanced solid tumors. J Clin Oncol 2005;23:3065.

32. Drew Y, Ledermann J, Hall G, et al. Phase 2 multicentre trial investigating intermittent and continuous dosing schedules of the poly(ADP-ribose) polymerase inhibitor rucaparib in germline BRCA mutation carriers with advanced ovarian and breast cancer. Br J Cancer 2016;114:723-30.

33. Kristeleit R, Shapiro GI, Burris HA, et al. A phase I-II study of the oral PARP inhibitor rucaparib in patients with germline BRCA1/2-mutated ovarian carcinoma or other solid tumors. Clin Cancer Res 2017;23:4095-106.

34. Swisher EM, Lin KK, Oza AM, et al. Rucaparib in relapsed, platinum-sensitive high-grade ovarian carcinoma (ARIEL2 Part 1): an international, multicentre, openlabel, phase 2 trial. Lancet Oncol 2017;18:75-87.

35. Burger RA, Brady MF, Bookman MA, et al. Incorporation of Bevacizumab in the Primary Treatment of Ovarian Cancer. N Engl J Med 2011;365:2473-83.

36. Aghajanian C, Blank SV, Goff BA, et al. OCEANS: A randomized, double-blind, placebo-controlled phase III trial of chemotherapy with or without bevacizumab in patients with platinum-sensitive recurrent epithelial ovarian, primary peritoneal, or fallopian tube cancer. J Clin Oncol 2012;30:2039-45.

37. Pujade-Lauraine E, Hilpert F, Weber B, et al. Bevacizumab combined with chemotherapy for platinum-resistant recurrent ovarian cancer: The AURELIA open-label randomized phase III trial. J Clin Oncol 2014;32:1302-8.

38. Ledermann JA, Embleton AC, Perren T, et al. Overall survival results of ICON6: A trial of chemotherapy and cediranib in relapsed ovarian cancer. J Clin Oncol 2017;35:5506.

39. Bindra RS, Gibson SL, Meng A, et al. Hypoxia-induced down-regulation of BRCA1 expression by E2Fs. Cancer Res 2005;65:11597-604.

40. Liu JF, Barry WT, Birrer M, et al. Combination cediranib and olaparib versus olaparib alone for women with recurrent platinum-sensitive ovarian cancer: A randomised phase 2 study. Lancet Oncol 2014;15:1207-14.

41. Liu JF, Barry WT, Birrer M, et al. Overall survival and updated progression-free survival outcomes in a randomized phase II study of combination cediranib and olaparib versus olaparib in relapsed platinum-sensitive ovarian cancer. Ann Oncol 2019;30:551-7.

42. Mirza MR, Åvall Lundqvist E, Birrer MJ, et al. Niraparib plus bevacizumab versus niraparib alone for platinumsensitive recurrent ovarian cancer (NSGO-AVANOVA2/ ENGOT-ov24): a randomised, phase 2, superiority trial. Lancet Oncol 2019;20:1409-19.

43. Ray-Coquard I, Pautier P, Pignata S, et al. Olaparib plus Bevacizumab as First-Line Maintenance in Ovarian 
Cancer. N Engl J Med 2019;381:2416-28.

44. LaFargue CJ, Dal Molin GZ, Sood AK, et al. Exploring and comparing adverse events between PARP inhibitors. Lancet Oncol 2019;20:e15-28.

45. Sanchez RI, Wang RW, Newton DJ, et al. Cytochrome $\mathrm{P} 4503 \mathrm{~A} 4$ is the major enzyme involved in the metabolism of the substance $\mathrm{P}$ receptor antagonist aprepitant. Drug Metab Dispos 2004;32:1287-92.

46. Friedlander M, Banerjee S, Mileshkin L, et al. Practical guidance on the use of olaparib capsules as maintenance therapy for women with BRCA mutations and platinumsensitive recurrent ovarian cancer. Asia Pac J Clin Oncol 2016;12:323-31.

47. Konstantinopoulos PA, Waggoner SE, Vidal GA, et al.

Cite this article as: Bahena-González A, Toledo-Leyva A, Gallardo-Rincón D. PARP inhibitors in ovarian cancer: evidence for maintenance and treatment strategies. Chin Clin Oncol 2020;9(4):51. doi: 10.21037/cco-20-69
TOPACIO/Keynote-162 (NCT02657889): A phase 1/2 study of niraparib + pembrolizumab in patients (pts) with advanced triple-negative breast cancer or recurrent ovarian cancer (ROC)—Results from ROC cohort. J Clin Oncol 2018;36:106.

48. Drew Y, de Jonge M, Hong SH, et al. An open-label, phase II basket study of olaparib and durvalumab (MEDIOLA): Results in germline BRCA -mutated (gBRCA m) platinumsensitive relapsed (PSR) ovarian cancer (OC). Gynecol Oncol 2018;149:246-7.

49. Coleman RL, Fleming GF, Brady MF, et al. Veliparib with First-Line Chemotherapy and as Maintenance Therapy in Ovarian Cancer. N Engl J Med 2019;381:2403-15. 